Document downloaded from:

http://hdl.handle.net/10251/125132

This paper must be cited as:

Mingueza, D.; Montoro, ME.; Roca Martinez, A. (2018). The lattice of characteristic subspaces of an endomorphism with Jordan-Chevalley decomposition. Linear Algebra and its Applications. 558:63-73. https://doi.org/10.1016/j.laa.2018.08.005

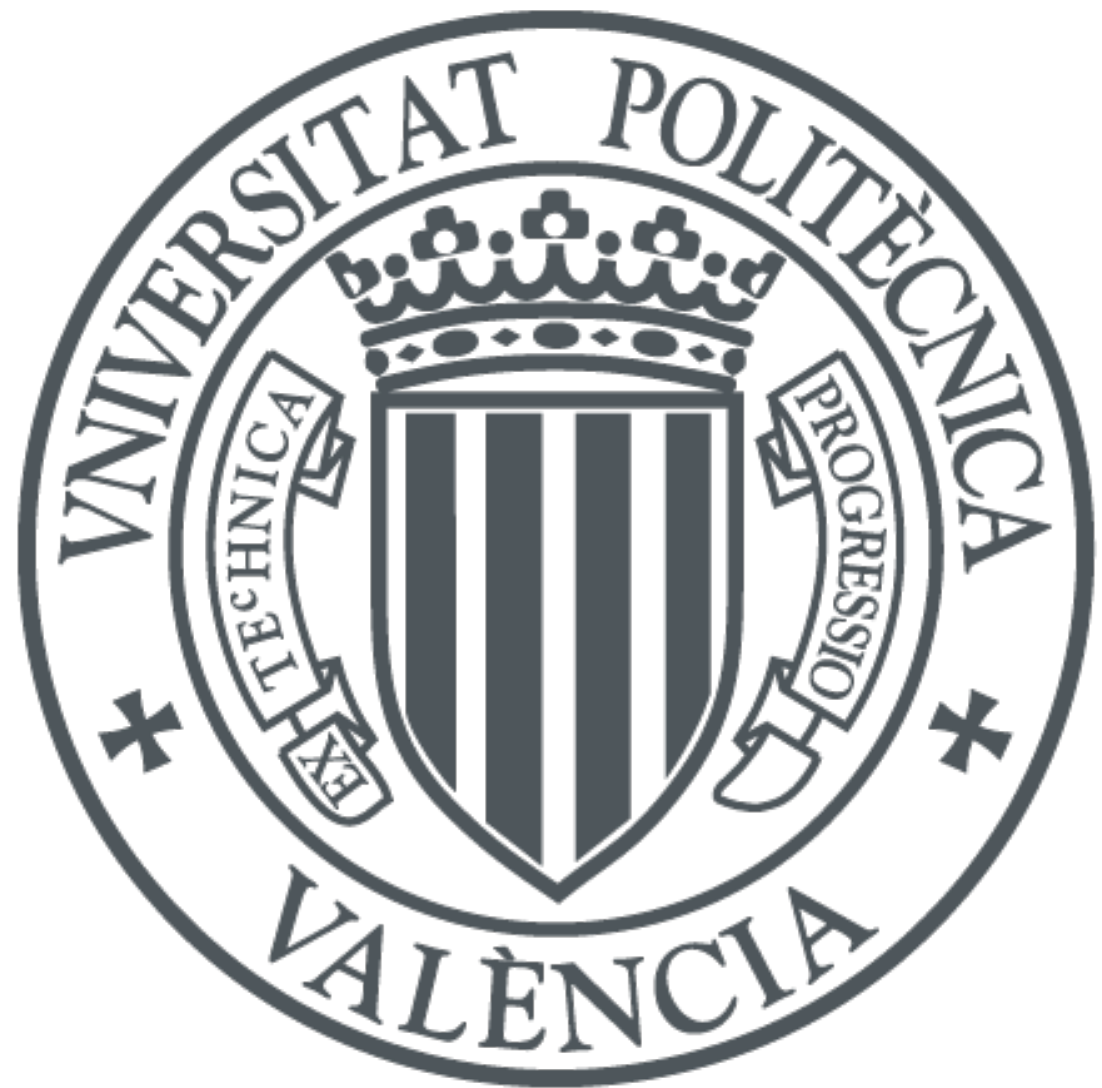

The final publication is available at

http://doi.org/10.1016/j.laa.2018.08.005

Copyright Elsevier

Additional Information 


\title{
The lattice of characteristic subspaces of an endomorphism with Jordan-Chevalley decomposition
}

\author{
David Mingueza \\ Accenture, 08022 Barcelona, Spain * \\ M. Eulàlia Montoro \\ Departamento de Matemáticas e Informática. Universitat de Barcelona ${ }^{\dagger}$ \\ Alicia Roca \\ Departamento de Matemática Aplicada, IMM, \\ Universitat Politècnica de València ${ }^{\ddagger}$
}

\begin{abstract}
Given an endomorphism $A$ over a finite dimensional vector space having Jordan-Chevalley decomposition, the lattices of invariant and hyperinvariant subspaces of $A$ can be obtained from the nilpotent part of this decomposition. We extend this result for lattices of characteristic subspaces. We also obtain a generalization of Shoda's Theorem about the characterization of the existence of characteristic non hyperinvariant subspaces.
\end{abstract}

Keywords: Hyperinvariant subspaces, characteristic subspaces, lattices.

\section{Introduction}

The lattice of characteristic subspaces of an endomorphism over a finite dimensional space has been studied in $[1,2,7,8]$, where structural properties of the lattice have been given when the minimal polynomial of the endomorphism splits over the underlying field $\mathbb{F}$. It was proved in ([1]) that only if $\mathbb{F}=G F(2)$, the lattices of characteristic and hyperinvariant subspaces may not coincide. When the minimal polynomial of the endomorphism does not split over $\mathbb{F}$, the lattice of characteristic subspaces has not been described. The aim of this paper

\footnotetext{
*david.mingueza@outlook.es

† eula.montoro@ub.edu

‡aroca@mat.upv.es
} 
is to analyze this case when the minimal polynomial of the endomorphism is separable.

The results are based on the Jordan-Chevalley decomposition of an endomorphism which exists if the minimal polynomial of the endomorphism is separable (see $[5,9])$. In particular, on perfect fields every irreducible polynomial is separable, therefore the Jordan-Chevalley decomposition holds.

The paper is organized as follows: in Section 2 we introduce some definitions and previous results. We present some lemmas showing that the study the lattices of the invariant and hyperinvariant subspaces of an endomorphism can be reduced to the case of an endomorphism where the minimal polynomial is a power of an irreducible polynomial $p$.

In Section 3, out of the Jordan-Chevalley decomposition of an endomorphism into its commuting semisimple and nilpotent parts, we reduce the problem to the study of the lattice of characteristic subspaces of the associated nilpotent part. Finally, we conclude that characteristic non hyperinvariant subspaces can only appear when the irreducible factor of the minimal polynomial is of degree 1 , in other words, if $p$ splits over $\mathbb{F}$. This result extends Shoda's theorem (Theorema 2.9) for the separable case.

\section{Preliminaries}

We introduce some definitions and previous results which will be used throughout the paper.

Let $\mathbb{F}$ be a field. Let $V$ be an $n$-dimensional vector space over $\mathbb{F}$ and $f$ : $V \rightarrow V$ an endomorphism. We denote by $A \in M_{n}(\mathbb{F})$ its associated matrix with respect to given basis and by $m_{A}$ the minimal polynomial of $A$. In what follows we will identify $f$ with $A$. The degree of a polynomial $p$ is written as $\operatorname{deg}(p)$, and $|\cdot|$ is the "cardinality of".

The lattice of the vector subspaces of $V$ will be denoted by $\mathrm{七}_{\mathbb{F}}(V)$.

A subspace $Y \subseteq V$ is invariant with respect to $A$ if $A Y \subseteq Y$. We denote by $\operatorname{Inv}_{\mathbb{F}}(A)$ the lattice of invariant subspaces.

The centralizer of $A$ over $\mathbb{F}$ is the algebra $Z_{\mathbb{F}}(A)=\left\{B \in M_{n}(\mathbb{F}): A B=\right.$ $B A\}$. If we only take those endomorphisms $B$ that are automorphisms we write $Z_{\mathbb{F}}^{*}(A)=\left\{B \in M_{n}(\mathbb{F}): A B=B A, \operatorname{det}(B) \neq 0\right\}$.

A subspace $Y \subseteq V$ is called hyperinvariant with respect to $A$ if $B Y \subseteq Y$ for all matrices $B \in \bar{Z}_{\mathbb{F}}(A)$. We denote by $\operatorname{Hinv}_{\mathbb{F}}(A)$ the lattice of hyperinvariant subspaces.

An invariant subspace $Y \subseteq V$ with respect to $A$ is called characteristic if $B Y \subseteq Y$ for all matrices $B \in Z_{\mathbb{F}}^{*}(A)$ and $A Y \subseteq Y$. We denote by $\operatorname{Chinv}_{\mathbb{F}}(A)$ the lattice of characteristic subspaces.

Obviously,

$$
\operatorname{Hinv}_{\mathbb{F}}(A) \subseteq \operatorname{Chinv}_{\mathbb{F}}(A) \subseteq \operatorname{Inv}_{\mathbb{F}}(A)
$$


The lattices of invariant and hyperinvariant subspaces allow the following decompositions.

Proposition 2.1. [3] Let $A$ and $B$ be endomorphisms on finite dimensional vector spaces $V$ and $W$ respectively, over a field $\mathbb{F}$. The following properties are equivalent:

1. The minimum polynomials of $A$ and $B$ are relatively prime.

2. $\operatorname{Inv}_{\mathbb{F}}(A \oplus B)=\operatorname{Inv}_{\mathbb{F}}(A) \oplus \operatorname{Inv}_{\mathbb{F}}(B)$.

Proposition 2.2. [4] Let $A$ and $B$ be endomorphisms on finite dimensional vector spaces $V$ and $W$ respectively, over a field $\mathbb{F}$. The following properties are equivalent:

1. The minimum polynomials of $A$ and $B$ are relatively prime.

2. $\operatorname{Hinv}_{\mathbb{F}}(A \oplus B)=\operatorname{Hinv}_{\mathbb{F}}(A) \oplus \operatorname{Hinv}_{\mathbb{F}}(B)$.

As a consequence, if $m_{A}=p_{1}^{k_{1}} \cdots p_{r}^{k_{r}}$ is the prime decomposition of $m_{A}$ for a given $A \in M_{n}(\mathbb{F})$, taking into account the primary decomposition

$$
V=V_{1} \oplus \cdots \oplus V_{r}, \quad V_{i}=\operatorname{ker}\left(p_{i}(A)^{k_{i}}\right), \quad i=1, \ldots, r,
$$

and $A_{i}=A \mid V_{i}, \quad i=1, \ldots r$, the next result is satisfied (see $[3,4]$ ).

Proposition 2.3. Let $A \in M_{n}(\mathbb{F})$ and $m_{A}=p_{1}^{k_{1}} \cdots p_{r}^{k_{r}}$ with $p_{1}, \ldots, p_{r}$ distinct irreducible polynomials. Then,

1. $\operatorname{Inv}_{\mathbb{F}}(A)=\operatorname{Inv}_{\mathbb{F}}\left(A_{1}\right) \oplus \cdots \oplus \operatorname{Inv}_{\mathbb{F}}\left(A_{r}\right)$.

2. $\operatorname{Hinv}_{\mathbb{F}}(A)=\operatorname{Hinv}_{\mathbb{F}}\left(A_{1}\right) \oplus \cdots \oplus \operatorname{Hinv}_{\mathbb{F}}\left(A_{r}\right)$.

If $p(x) \in \mathbb{F}[x]$ is an irreducible polynomial and $\alpha \notin \mathbb{F}$ is a root of $p(x)$, we denote by $\mathbb{F}(\alpha)$ the minimal extension of $\mathbb{F}$ containing $\alpha$. The next result can be found in [3]. Although a proof of the following property 4 was included in [3], we give here a simple proof of the whole lemma.

Lemma 2.4. [3] Let $A \in M_{n}(\mathbb{F})$. Assume that $m_{A}=p_{0}+p_{1} x+\ldots+p_{s} x^{s} \in \mathbb{F}[x]$ is irreducible, and that $\alpha$ is a root of $p$ such that $\alpha \notin \mathbb{F}$. Let $\mathbb{K}=\left\{a_{0} I_{n}+a_{1} A+\right.$ $\left.\ldots+a_{s-1} A^{s-1} \mid a_{i} \in \mathbb{F}\right\}$ be the algebra of polynomials of degree at most $s$. Then,

1. $\mathbb{K}$ is a field isomorphic to $\mathbb{F}(\alpha)$.

2. $V$ is a $\mathbb{K}$-vector space.

3. $A$ is $\mathbb{K}$-linear.

4. $\operatorname{Inv}_{\mathbb{F}}(A)=E_{\mathbb{K}}(V)$. 
Proof. 1. The following application

$$
\Gamma: \mathbb{K} \longrightarrow \mathbb{F}(\alpha),
$$

with $\Gamma\left(a_{0} I_{n}+a_{1} A \ldots+a_{s-1} A^{s-1}\right)=a_{0}+a_{1} \alpha+\ldots+a_{s-1} \alpha^{s-1}$ is an isomorphism.

2. For all $\lambda=a_{0} I_{n}+a_{1} A+\ldots+a_{s-1} A^{s-1} \in \mathbb{K}$, it is satisfied that

$$
\lambda v=\left(a_{0} I_{n}+a_{1} A+\ldots+a_{s-1} A^{s-1}\right) v \in V, \quad \forall v \in V .
$$

3. For all $\lambda \in \mathbb{K}, A \lambda v=\lambda A v$.

4. The following equivalences hold,

$$
W \in \operatorname{Inv}_{\mathbb{F}}(A) \Leftrightarrow A W \subseteq W \Leftrightarrow \lambda W \subseteq W, \forall \lambda \in \mathbb{K} \Leftrightarrow W \in \mathrm{L}_{\mathbb{K}}(V) .
$$

Notice that properties $1-4$ of this lemma are trivially true if $s=1$.

A polynomial is called p-primary if it is of the form $p^{r}$ for some irreducible polynomial $p$ and a positive integer $r$. A polynomial $p(x)$ over a field $\mathbb{F}$ is called separable if its roots are distinct in an algebraic closure of $\mathbb{F}$.

A linear operator $\mathrm{S}$ on a finite-dimensional vector space is semisimple if every S-invariant subspace has a complementary S-invariant subspace. A linear operator $\mathrm{N}$ on a finite-dimensional vector space is nilpotent if $N^{k}=0$ for some positive integer $\mathrm{k}$.

The next lemma contains the Jordan-Chevalley decomposition of a matrix, which plays a key role in this work. The result can be found in [9].

Lemma 2.5. (Jordan-Chevalley decomposition) Let $A \in M_{n}(\mathbb{F})$ be a matrix with $m_{A}=p^{r}$, where $p$ is irreducible and separable. Then, there is a unique decomposition

$$
A=S+N,
$$

where $S$ is semisimple, $N$ is nilpotent and $S N=N S$. Moreover, $S$ and $N$ are polynomials in $A$.

Remark 2.6. [3]

If $A$ has Jordan-Chevalley decomposition then there exist a transformation into a rational canonical form such that $A$ is similar to

$$
\operatorname{diag}\left(C_{1}, C_{2}, \ldots, C_{m}\right),
$$

with

$$
C_{i}=\left(\begin{array}{ccccc}
C & 0 & \ldots & 0 & 0 \\
I_{s} & C & \ldots & 0 & 0 \\
\vdots & \vdots & \ddots & \vdots & \vdots \\
0 & 0 & \ldots & C & 0 \\
0 & 0 & \ldots & I_{s} & C
\end{array}\right) \in M_{n_{i}}(\mathbb{F}), \quad i=1,2, \ldots, m
$$


where $C$ is the companion matrix of $p$, and $n_{1} \geq n_{2} \geq \cdots \geq n_{m}>0, n_{1}+\ldots+$ $n_{m}=n, n_{1}=s r($ where $s=\operatorname{deg}(p))$, then

$$
S=\operatorname{diag}\left(S_{1}, S_{2}, \ldots, S_{m}\right), \quad N=\operatorname{diag}\left(N_{1}, N_{2}, \ldots, N_{m}\right),
$$

where

$$
S_{i}=\left(\begin{array}{ccccc}
C & 0 & \ldots & 0 & 0 \\
0 & C & \ldots & 0 & 0 \\
\vdots & \vdots & \ddots & \vdots & \vdots \\
0 & 0 & \ldots & C & 0 \\
0 & 0 & \ldots & 0 & C
\end{array}\right), \quad N_{i}=\left(\begin{array}{ccccc}
0 & 0 & \ldots & 0 & 0 \\
I_{s} & 0 & \ldots & 0 & 0 \\
\vdots & \vdots & \ddots & \vdots & \vdots \\
0 & 0 & \ldots & 0 & 0 \\
0 & 0 & \ldots & I_{s} & 0
\end{array}\right) .
$$

Taking advantage of the Jordan-Chevalley decomposition it can be proved that the lattices of the invariant and hyperinvariant subspaces of $A$ over $\mathbb{F}$ can be obtained as lattices of the corresponding subspaces of a nilpotent matrix over a different field $\mathbb{K}$ (see [3] and [4], respectively). The results are included in the next theorem. Although proofs are sketched in [3, 4], we provided them in detail.

Theorem 2.7. Let $A \in M_{n}(\mathbb{F})$. Assume that $m_{A}$ is $p$-primary $\left(m_{A}=p^{r}\right)$ with $p$ separable and $\operatorname{deg}(p)=s$. Let $A=S+N$ be the Jordan-Chevalley decomposition of $A$. Let $\mathbb{K}=\left\{a_{0} I_{n}+a_{1} S+\ldots+a_{s-1} S^{s-1} \mid a_{i} \in \mathbb{F}\right\}$. Then,

1. $\operatorname{Inv}_{\mathbb{F}}(A)=\operatorname{Inv}_{\mathbb{K}}(A)=\operatorname{Inv}_{\mathbb{K}}(N)$.

2. $\operatorname{Hinv}_{\mathbb{F}}(A)=\operatorname{Hinv}_{\mathbb{K}}(A)=\operatorname{Hinv}_{\mathbb{K}}(N)$.

Proof. 1. $W \in \operatorname{Inv}_{\mathbb{F}}(A) \Leftrightarrow W \in \operatorname{Inv}_{\mathbb{K}}(A)$ (because $A$ is $\mathbb{K}$ - linear). Moreover, since $S \in \mathbb{K}$

$$
\operatorname{Inv}_{\mathbb{K}}(A)=\operatorname{Inv}_{\mathbb{K}}(A-S)=\operatorname{Inv}_{\mathbb{K}}(N) .
$$

2. Notice that

$$
Z_{\mathbb{K}}(A)=Z_{\mathbb{F}}(A) \cap Z_{\mathbb{F}}(S)=Z_{\mathbb{F}}(A),
$$

and

$$
Z_{\mathbb{K}}(A)=Z_{\mathbb{K}}(A-S)=Z_{\mathbb{K}}(N)
$$

Therefore

$$
\operatorname{Hinv}_{\mathbb{F}}(A)=\operatorname{Hinv}_{\mathbb{K}}(A)=\operatorname{Hinv}_{\mathbb{K}}(A-S)=\operatorname{Hinv}_{\mathbb{K}}(N) .
$$

Next example illustrates the above lemma for the field of real numbers.

Example 2.8. Let $\mathbb{F}=\mathbb{R}, V=\mathbb{R}^{4}$ and

$$
A=\left(\begin{array}{cccc}
0 & 1 & 0 & 0 \\
-1 & 0 & 0 & 0 \\
1 & 0 & 0 & 1 \\
0 & 1 & -1 & 0
\end{array}\right) .
$$


The minimal polynomial of $A$ is $m_{A}=\left(x^{2}+1\right)^{2}$. The Jordan-Chevalley decomposition of $A$ is given by

$$
S=\left(\begin{array}{cccc}
0 & 1 & 0 & 0 \\
-1 & 0 & 0 & 0 \\
0 & 0 & 0 & 1 \\
0 & 0 & -1 & 0
\end{array}\right), \quad N=\left(\begin{array}{llll}
0 & 0 & 0 & 0 \\
0 & 0 & 0 & 0 \\
1 & 0 & 0 & 0 \\
0 & 1 & 0 & 0
\end{array}\right)
$$

and $\mathbb{K}=\left\{a_{0} I_{4}+a_{1} S, a_{i} \in \mathbb{R}\right\}$. Notice that the Jordan chains of $N$ are

$$
\begin{aligned}
& e_{1} \rightarrow e_{3} \rightarrow 0, \\
& e_{2} \rightarrow e_{4} \rightarrow 0 .
\end{aligned}
$$

Then,

$$
\operatorname{Inv}_{\mathbb{R}}(A)=\operatorname{Inv}_{\mathbb{K}}(N)=\left\{0,\left\langle e_{3}, e_{4}\right\rangle, V\right\} .
$$

The lattice of hyperinvariant subspaces is

$$
\operatorname{Hinv}_{\mathbb{R}}(A)=\operatorname{Hinv}_{\mathbb{K}}(N)=\left\{0,\left\langle e_{3}, e_{4}\right\rangle, V\right\} .
$$

Concerning to characteristic subspaces, Shoda's theorem characterizes the existence of characteristic non hyperinvariant subspaces.

Theorem 2.9. [10] Let $V$ be a finite-dimensional vector space over the field $\mathbb{F}=G F(2)$ and let $f: V \rightarrow V$ be a nilpotent linear operator. The following statements are equivalent:

1. There exists a characteristic subspace of $V$ which is not hyperinvariant.

2. For some numbers $r$ and $s$ with $s>r+1$ the Jordan form of $f$ contains exactly one Jordan block of size $s$ and exactly one block of size $r$.

Astuti-Wimmer proved ([1]) that characteristic non hyperinvariant subspaces can only exist on the field $G F(2)$.

Theorem 2.10. [1] Let $V$ be a finite dimensional vector space over a field $\mathbb{F}$ and let $f: V \rightarrow V$ be a linear operator. Assume that the minimal polynomial of $f$ splits over $\mathbb{F}$. If $|\mathbb{F}|>2$, then $\operatorname{Chinv}_{\mathbb{F}}(f)=\operatorname{Hinv}_{\mathbb{F}}(f)$.

\section{Reduction to the nilpotent case for character- istic subspaces}

In this section we focus on the study of the lattice of characteristic subspaces of an endomorphism over a field when the irreducible factors of the minimal polynomial are separable.

First of all we see that the general case can be reduced to the especific case of endomorphisms having minimal polynomials with an unique irreducible factor, as in the lattices of invariant of hyperinvariant subspaces. 
Lemma 3.1. Let $A$ and $B$ be endomorphisms on finite dimensional vector spaces $V$ and $W$ respectively, over a field $\mathbb{F}$. The following properties are equivalent:

1. The minimum polynomials of $A$ and $B$ are relatively prime.

2. $\operatorname{Chinv}_{\mathbb{F}}(A \oplus B)=\operatorname{Chinv}_{\mathbb{F}}(A) \oplus \operatorname{Chinv}_{\mathbb{F}}(B)$.

Proof. First assume that (1) is true. It is evident that $\operatorname{Chinv}(A \oplus B) \subseteq \operatorname{Chinv}(A) \oplus$ Chinv $(B)$ holds even for an arbitrary polynomials.

As it is stated in [11], $Z(A \oplus B)=Z(A) \oplus Z(B)$, therefore every commuting automorphism of $A \oplus B$ is of the form $X=\left(\begin{array}{cc}X_{1} & 0 \\ 0 & X_{2}\end{array}\right)$ with $X_{1} \in Z^{*}(A)$ and $X_{2} \in Z^{*}(B)$.

Taking $W=W_{1} \oplus W_{2} \in \operatorname{Chinv}(A) \oplus \operatorname{Chinv}(B)$, clearly $W \in \operatorname{Chinv}(A \oplus B)$ follows.

Assume now that (2) is true.

As $\operatorname{Chinv}(A \oplus B)=\operatorname{Chinv}(A) \oplus \operatorname{Chinv}(B)$, it is satisfied that $V \oplus 0 \in$ $\operatorname{Chinv}(A \oplus B)$. Notice that if $X$ is a matrix such that $X A=B X$, then $\left(\begin{array}{cc}I & 0 \\ X & I\end{array}\right) \in Z^{*}(A \oplus B)$. Therefore, $V \oplus 0$ must be invariant for this matrix and this implies $X=0$.

If $\operatorname{gcd}\left(m_{A}, m_{B}\right)=q, \operatorname{deg}(q)>0$, then $m_{A}=q f$ and $m_{B}=q h$ for some polynomials $f, h$, and w.l.o.g. we can $\operatorname{suppose} \operatorname{gcd}(q, f)=1$. With respect to an appropriate basis, we can write $A=\left(\begin{array}{cc}C_{q} & 0 \\ 0 & F\end{array}\right)$ and $B=\left(\begin{array}{cc}C_{q} & H \\ 0 & G\end{array}\right)$ where $C_{q}$ is the companion matrix of $q$ and for some matrices $F, H$ and $G$.

Let $U \in Z^{*}\left(C_{q}\right)$, then $X=\left(\begin{array}{cc}U & 0 \\ 0 & 0\end{array}\right) \neq 0$ satisfies $X A=B X$, which is clearly a contradiction.

Corollary 3.2. Let $A \in M_{n}(\mathbb{F})$ and $m_{A}=p_{1}^{k_{1}} \ldots p_{r}^{k_{r}}$ with $p_{i}$ distinct irreducible polynomials. Then,

$$
\operatorname{Chinv}(A)=\operatorname{Chinv}\left(A_{1}\right) \oplus \cdots \oplus \operatorname{Chinv}\left(A_{r}\right)
$$

where $A_{i} \in M_{n_{i}}(\mathbb{F})$ is the restriction of $A \in M_{n}(\mathbb{F})$ to $V_{i}=\operatorname{ker}\left(\left(p_{i}(A)\right)^{k_{i}}\right)$, $i=1,2, \ldots, r,\left(V=V_{1} \oplus \cdots \oplus V_{r}\right)$.

In the next lemma we see that the lattice of characteristic subspaces of $A$ can also be determined on a different field for a nilpotent matrix, using JordanChevalley decomposition.

Lemma 3.3. Let $A \in M_{n}(\mathbb{F})$. Assume that $m_{A}$ is $p$-primary $\left(m_{A}=p^{r}\right)$ with $p$ separable and $\operatorname{deg}(p)=s$. Let $A=S+N$ be the Jordan-Chevalley decomposition of $A$. Let $\mathbb{K}=\left\{a_{0} I_{n}+a_{1} S+\ldots+a_{s-1} S^{s-1} \mid a_{i} \in \mathbb{F}\right\}$. Then,

1. $\operatorname{Chinv}_{\mathbb{F}}(A)=\operatorname{Chinv}_{\mathbb{K}}(A)=\operatorname{Chinv}_{\mathbb{K}}(N)$. 
Proof. Similarly to the proof of Theorem 2.7,

$$
Z_{\mathbb{K}}^{*}(A)=Z_{\mathbb{F}}^{*}(A) \cap Z_{\mathbb{F}}^{*}(S)=Z_{\mathbb{F}}^{*}(A),
$$

and

Therefore

$$
Z_{\mathbb{K}}^{*}(A)=Z_{\mathbb{K}}^{*}(A-S)=Z_{\mathbb{K}}^{*}(N)
$$

$\operatorname{Chinv}_{\mathbb{F}}(A)=\operatorname{Chinv}_{\mathbb{K}}(A)=\operatorname{Chinv}_{\mathbb{K}}(A-S)=\operatorname{Chinv}_{\mathbb{K}}(N)$.

Finally we obtain the conclusion that characteristic non hyperinvariant subspaces can only exist over Jordan blocks.

Theorem 3.4. Let $A$ be an endomorphism on a finite dimensional space $V$ over a field $\mathbb{F}$. Assume that $m_{A}$ is $p$-primary $\left(m_{A}=p^{r}\right)$ with $p$ separable. If $\operatorname{Chinv}_{\mathbb{F}}(A) \backslash \operatorname{Hinv}_{\mathbb{F}}(A) \neq \emptyset$, then $\operatorname{deg}(p)=1$ and $\mathbb{F}=G F(2)$.

Proof. Let us assume that $\operatorname{Chinv}_{\mathbb{F}}(A) \backslash \operatorname{Hinv}_{\mathbb{F}}(A) \neq \emptyset$ and $\operatorname{deg}(p)>1$. As $p$ is separable, let $A=N+S$ be the Jordan-Chevalley decomposition of $A$. By Lemma 3.3 we know that $\operatorname{Chinv}_{\mathbb{F}}(A)=\operatorname{Chinv}_{\mathbb{K}}(N)$ with $\mathbb{K}$ defined as in Lemma 3.3. By assumption $\operatorname{deg}(p)>1$, which implies that $|\mathbb{K}|>2$, and by $[1$, Theorem 3.4], $\operatorname{Chinv}_{\mathbb{K}}(N)=\operatorname{Hinv}_{\mathbb{K}}(N)$. Finally, by Theorem 2.7, $\operatorname{Hinv}_{\mathbb{K}}(N)=\operatorname{Hinv}_{\mathbb{F}}(A)$. Therefore, $\operatorname{Chinv}_{\mathbb{F}}(A)=\operatorname{Hinv}_{\mathbb{F}}(A)$, which is a contradiction.

We see that for the existence of characteristic non-hyperinvariant subspaces it is necessary that $\operatorname{deg}(p)=1$ and $\mathbb{F}=G F(2)$. If we take into account the Shoda condition, we obtain a necessary and sufficient condition.

Corollary 3.5. Let $A$ be an endomorphism on a finite dimensional space $V$ over a field $\mathbb{F}$. Assume that $m_{A}$ is $p$-primary $\left(m_{A}=p^{r}\right)$ with $p$ separable. Then, the next two statement are equivalent:

1. $\operatorname{Chinv}_{\mathbb{F}}(A) \backslash \operatorname{Hinv}_{\mathbb{F}}(A) \neq \emptyset$.

2. $\mathbb{F}=G F(2), \operatorname{deg}(p)=1$ and the Shoda condition is satisfied.

We show next how the lattice of characteristic subspaces can be obtained in two examples. In the first one $\operatorname{Hinv}_{\mathbb{F}}(A)=\operatorname{Chinv}_{\mathbb{F}}(A)$, and in the second one $\operatorname{Chinv}_{\mathbb{F}}(A) \nsubseteq \operatorname{Hinv}_{\mathbb{F}}(A)$ for some matrices $A$.

Example 3.6. Let $\mathbb{F}=G F(2)$ and

$$
A=\left(\begin{array}{llllll|ll}
0 & 1 & 0 & 0 & 0 & 0 & 0 & 0 \\
1 & 1 & 0 & 0 & 0 & 0 & 0 & 0 \\
1 & 0 & 0 & 1 & 0 & 0 & 0 & 0 \\
0 & 1 & 1 & 1 & 0 & 0 & 0 & 0 \\
0 & 0 & 1 & 0 & 0 & 1 & 0 & 0 \\
0 & 0 & 0 & 1 & 1 & 1 & 0 & 0 \\
\hline 0 & 0 & 0 & 0 & 0 & 0 & 0 & 1 \\
0 & 0 & 0 & 0 & 0 & 0 & 1 & 1
\end{array}\right) .
$$


The minimal polynomial of $A$ is $p$-primary, $m_{A}=\left(x^{2}+x+1\right)^{3}, p=x^{2}+x+1$ separable, and the Jordan-Chevalley decomposition of $A$ is

$$
S=\left(\begin{array}{llllll|ll}
0 & 1 & 0 & 0 & 0 & 0 & 0 & 0 \\
1 & 1 & 0 & 0 & 0 & 0 & 0 & 0 \\
0 & 0 & 0 & 1 & 0 & 0 & 0 & 0 \\
0 & 0 & 1 & 1 & 0 & 0 & 0 & 0 \\
0 & 0 & 0 & 0 & 0 & 1 & 0 & 0 \\
0 & 0 & 0 & 0 & 1 & 1 & 0 & 0 \\
\hline 0 & 0 & 0 & 0 & 0 & 0 & 0 & 1 \\
0 & 0 & 0 & 0 & 0 & 0 & 1 & 1
\end{array}\right), \quad N=\left(\begin{array}{llllll|ll}
0 & 0 & 0 & 0 & 0 & 0 & 0 & 0 \\
0 & 0 & 0 & 0 & 0 & 0 & 0 & 0 \\
1 & 0 & 0 & 0 & 0 & 0 & 0 & 0 \\
0 & 1 & 0 & 0 & 0 & 0 & 0 & 0 \\
0 & 0 & 1 & 0 & 0 & 0 & 0 & 0 \\
0 & 0 & 0 & 1 & 0 & 0 & 0 & 0 \\
\hline 0 & 0 & 0 & 0 & 0 & 0 & 0 & 0 \\
0 & 0 & 0 & 0 & 0 & 0 & 0 & 0
\end{array}\right) .
$$

Let $\mathbb{K}=\left\{a_{0} I_{8}+a_{1} S, a_{i} \in G F(2)\right\}$.

The Segre characteristic of $N$ is $(3,3,1,1)$ with Jordan chains

$$
\begin{aligned}
& e_{1} \rightarrow e_{3} \rightarrow e_{5} \rightarrow 0 \\
& e_{2} \rightarrow e_{4} \rightarrow e_{6} \rightarrow 0 \\
& e_{7} \rightarrow 0 \\
& e_{8} \rightarrow 0
\end{aligned}
$$

The characteristic and hyperinvariant subspaces of $N$ are (see [7]):

$\operatorname{Hinv}_{\mathbb{K}}(N)=\operatorname{Chinv}_{\mathbb{K}}(N)=\left\{0,\left\langle e_{3}, e_{5}, e_{4}, e_{6}, e_{7}, e_{8}\right\rangle,\left\langle e_{3}, e_{5}, e_{4}, e_{6}\right\rangle,\left\langle e_{5}, e_{6}, e_{7}, e_{8}\right\rangle,\left\langle e_{5}, e_{6}\right\rangle, V\right\}$.

Example 3.7. Let $\mathbb{F}=G F(2)$ and

$$
A=\left(\begin{array}{llll}
1 & 0 & 0 & 0 \\
1 & 1 & 0 & 0 \\
0 & 1 & 1 & 0 \\
0 & 0 & 0 & 1
\end{array}\right) .
$$

The minimal polynomial of $A$ is $m_{A}=(x+1)^{3}, p=x+1$ trivially splits over $\mathbb{F}$ and

$$
S=\left(\begin{array}{llll}
1 & 0 & 0 & 0 \\
0 & 1 & 0 & 0 \\
0 & 0 & 1 & 0 \\
0 & 0 & 0 & 1
\end{array}\right), \quad N=\left(\begin{array}{cccc}
0 & 0 & 0 & 0 \\
1 & 0 & 0 & 0 \\
0 & 1 & 0 & 0 \\
0 & 0 & 0 & 0
\end{array}\right)
$$

Observe that $\mathbb{K}=\mathbb{F}=G F(2)$ as $\operatorname{deg}(p)=1$.

The Segre characteristic of $N$ is $(3,1)$ with vector chains:

$$
\begin{aligned}
& e_{1} \rightarrow e_{2} \rightarrow e_{3} \rightarrow 0 \\
& e_{4} \rightarrow 0
\end{aligned}
$$

The hyperinvariant and characteristic non hyperinvariant subspaces, according to [7], are:

$$
\operatorname{Hinv}_{\mathbb{K}}(N)=\left\{0,\left\langle e_{2}, e_{3}, e_{4}\right\rangle,\left\langle e_{2}, e_{3}\right\rangle,\left\langle e_{3}, e_{4}\right\rangle,\left\langle e_{3}\right\rangle, V\right\},
$$

$$
\operatorname{Chinv}_{\mathbb{K}}(N) \backslash \operatorname{Hinv}_{\mathbb{K}}(N)=\left\{e_{2}+e_{4}+\left\langle e_{3}\right\rangle\right\} .
$$




\section{Acknowledgments}

The second author is partially supported by grant MTM2015-65361-P MINECO/FEDER, UE and MTM2017-90682-REDT. The third author is partially supported by grants MTM2017-83624-P and MTM2017-90682-REDT.

\section{References}

[1] P. Astuti, H.K. Wimmer. Hyperinvariant, characteristic and marked subspaces. Oper. Matrices 3, (2009) 261-270.

[2] P. Astuti, H.K. Wimmer. Characteristic and hyperinvariant subspaces over the field GF(2). Linear Algebra Appl. 438, 4, (2013), 1551-1563.

[3] L. Brickman, P.A. Fillmore. The invariant subspace lattice of a linear transformation. Can. J. Math., 19, 35, (1967), 810-822.

[4] P.A. Fillmore, D.A. Herrero and W.E. Longstaff. The hyperinvariant subspace lattice of a linear transformation. Linear Algebra Appl. 17 (1977), 125-132.

[5] K.Hoffman and R. Kunze. Linear Algebra. Second Edition, Prentice-Hall, Englewood Cliffs, N.J.,1971.

[6] 2002]Lang02S. Lang. Algebra, Vol. I, Princeton, N.J., 1953.

[7] D. Mingueza, M.E. Montoro, J.R. Pacha. Description of the characteristic non-hyperinvariant subspaces over the field $G F(2)$. Linear Algebra Appl. 439 (2013), 3734-3745.

[8] D. Mingueza, M.E. Montoro, A. Roca. The characteristic subspace lattice of a linear transformation. Linear Algebra Appl. 506 (2016) 329-341.

[9] C. Norman. Finitely Generated Abelian Groups and Similarity of Matrices over a Field. Springer-Verlag, London Ltd. 2012.

[10] K. Shoda. Über die characteristischen Untergruppen einer endlichen Abelschen Gruppe. Math. Z. 31 (1930) 611-624.

[11] D. A. Suprunenko,R. I. Tyshkevich. Commutative Matrices. Academic Paperbacks, 1968 . 